Arq. Bras. Med. Vet. Zootec., v.70, n.5, p.1557-1564, 2018

\title{
Refrigeração do sêmen da garoupa-verdadeira Epinephelus marginatus
}

\author{
[Refrigerated storage of dusky grouper Epinephelus marginatus sperm] \\ F.C. Silva, V.V. Kuhnen, E.G. Sanches* \\ Instituto de Pesca - Ubatuba, SP
}

\begin{abstract}
RESUMO
Foram estudados diferentes diluentes no processo de refrigeração do sêmen da garoupa-verdadeira. Inicialmente, a taxa e a duração da motilidade e a concentração espermática foram avaliadas, caracterizando a qualidade do sêmen fresco. Para os testes de refrigeração a $4^{\circ} \mathrm{C}$, diferentes diluentes foram testados em atmosfera normal e modificada (100\% oxigênio). O sêmen fresco apresentou concentração espermática de $3,1 \pm 0,2 \times 10^{9}$ células $\mathrm{mL}^{-1}$, motilidade média de $90 \%$, permanecendo móvel, em média, por 3.060 segundos. No experimento de refrigeração, a taxa e a duração média da motilidade foram mantidas adequadas durante 144 horas para o diluente A $(70 \% ; 3100$ segundos) em atmosfera normal. Na atmosfera modificada, a qualidade do sêmen caiu drasticamente durante as primeiras 24 horas, independentemente do diluente empregado, não propiciando vantagem em sua utilização. A refrigeração do sêmen da garoupa-verdadeira permite manter por até 144 horas uma apropriada qualidade espermática.
\end{abstract}

Palavras-chave: diluentes, atmosfera modificada, resfriamento espermático, espermatozoides

\begin{abstract}
This study aimed to evaluate different extenders in refrigerated storage of dusky grouper sperm. Initially, motility rate, motility time, and sperm density were analyzed to characterize the fresh sperm quality. Different diluents were used in the refrigerated storage sperm at $4^{\circ} \mathrm{C}$ in normal atmosphere and modified atmosphere (100\% oxygen). The fresh sperm has a spermatozoa density of $3.1 \pm 0.2 \times 10^{9}$ spermatozoa $\mathrm{mL}^{-}$ ${ }^{1}$, motility rate $90 \%$ and motility time of 3,060 seconds. In the experiment of refrigerated storage, the motility rate and the motility time were maintained appropriate for 144 hours for the extender A $(70 \%$; 3,100 seconds) in normal atmosphere. In the modified atmosphere, the sperm quality decreased drastically during the first 24 hours, independently of the diluent used, not propitiating advantages. The refrigerated sperm of dusky grouper is a viable alternative for the short-term storage, being possible, to maintain for up to 144 hours an appropriate sperm viability.
\end{abstract}

Keywords: extenders, modified atmosphere, spermatic cooling, spermatozoid

\section{INTRODUÇÃO}

A piscicultura marinha de espécies ameaçadas pode ser desenvolvida como um instrumento estratégico de conservação, além de, paralelamente, fomentar o desenvolvimento de uma nova cadeia produtiva, beneficiando a expansão da maricultura e a geração de emprego e renda para comunidades litorâneas (Sanches et al., 2009). A garoupa-verdadeira Epinephelus marginatus (Lowe, 1834) é uma espécie hermafrodita protogínica, em que as fêmeas

Recebido em 25 de julho de 2017

Aceito em 17 de novembro de 2017

*Autor para correspondência (corresponding author)

E-mail: esanches@pesca.sp.gov.br maturam com $2,5 \mathrm{~kg}$ e $38 \mathrm{~cm}$ (cinco anos de idade) e invertem o sexo com $11,0 \mathrm{~kg}$ e $57 \mathrm{~cm}$ (sete anos de idade), apresentando baixa resiliência e alta vulnerabilidade à exploração humana (Cunha et al., 2013). Atualmente está incluída como ameaçada na lista vermelha da International Union for Conservation of Nature and Natural Resources (Red..., 2012). No caso da garoupa-verdadeira, significativos avanços científicos já foram obtidos na área de patologia (Roumbedakis et al., 2013; Souza et al., 2014) e em técnicas de manejo alimentar (Ramos et al., 2012; Sanches et al., 2014). Entretanto, no 
campo da reprodução, ainda existem lacunas para a produção de formas jovens em cativeiro (Cunha et al., 2013).

Importante ferramenta para a reprodução de peixes, a refrigeração do sêmen tem contribuído para o sucesso da propagação artificial de inúmeras espécies (Sanches e Cerqueira, 2010). Essa técnica tem como objetivo manter a viabilidade espermática por um curto período de tempo (horas ou dias) em temperaturas de refrigeração $\left(4^{\circ} \mathrm{C}\right)$. A partir daí, o sêmen pode ser utilizado posteriormente no processo de fertilização, dispensando a presença dos machos no ato da fecundação e liberando tempo para o monitoramento da ovulação das fêmeas, o que proporciona melhores resultados nos procedimentos de reprodução (Oliveira et al., 2007). A utilização de técnicas de conservação de sêmen é essencial para os procedimentos de reprodução de espécies hermafroditas protogínicas (Kiriyakit et al., 2011). Apesar de sua importância, ainda são escassos os estudos de aplicação dessa técnica na reprodução de peixes de água salgada (Sanches e Cerqueira, 2011). A produção de formas jovens da garoupaverdadeira vem sendo realizada por empreendimentos privados (Kerber et al., 2012), porém ainda não existem relatos na literatura sobre a refrigeração do sêmen dessa espécie.

Para a refrigeração do sêmen, faz-se necessária a utilização de diluentes, que têm a função de fornecer um meio protetor aos espermatozoides. A diluição possibilita reduzir a competição entre os espermatozoides por oxigênio e espaço e pode, paralelamente, evitar a iniciação da motilidade espermática (Sanches et al., 2009). Por outro lado, o desenvolvimento de diluentes espécie-específicos pode propiciar melhores resultados na preservação da viabilidade espermática, auxiliando na padronização das técnicas de refrigeração e congelamento de sêmen de peixes (Salmito-Vanderley et al., 2012). Alguns autores consideram essencial uma expressiva disponibilidade de oxigênio para os espermatozoides quando o sêmen é submetido ao processo de refrigeração e indicam manter o sêmen em uma atmosfera exclusiva de oxigênio durante esse processo (Billard et al., 2004). Considerando-se os resultados positivos obtidos no congelamento do sêmen da garoupaverdadeira, no presente estudo serão utilizadas as mesmas três soluções iônicas anteriormente utilizadas com sucesso na criopreservação de sêmen dessa espécie (Sanches et al., 2009). A hipótese aqui levantada é a de que esses diluentes deverão proporcionar resultado distinto no processo de refrigeração, visto o diferente gradiente térmico utilizado.

O objetivo deste trabalho foi avaliar o efeito de diferentes diluentes, em atmosfera normal e modificada ( $100 \%$ oxigênio), na preservação de sêmen refrigerado da garoupa-verdadeira $E$. marginatus.

\section{MATERIAL E MÉTODOS}

Dez exemplares de garoupa-verdadeira (Epinephelus marginatus), coletadas na região litorânea de Ubatuba/SP (Sisbio 15.080-1), foram invertidos sexualmente (Sanches et. al., 2009) e mantidos em três tanques circulares com 3.000 litros, em sistema de recirculação de água salgada, dotado de filtragem mecânica (bag de $100 \mu \mathrm{m})$, skimmer e esterilização da água por meio de lâmpadas ultravioleta. A temperatura foi mantida constante $\left(28 \pm 1,0^{\circ} \mathrm{C}\right.$, controlada por termostatos e aquecedores). Os peixes foram alimentados uma vez ao dia, com sardinha Sardinella brasiliensis (Steindachner, 1879) até a saciação aparente.

Os peixes $(44,5 \pm 3,7 \mathrm{~cm}$ e $1377,9 \pm 357,7 \mathrm{~g})$, após 24 horas de jejum, foram anestesiados (benzocaína a $0,1 \mathrm{~g} / \mathrm{L}$ ), e, em seguida, o sêmen foi extraído, por extrusão, individualmente de cada exemplar (sem a necessidade de indução hormonal), e coletado em uma seringa plástica para insulina graduada $(1 \mathrm{~mL})$ envolvida com papel-alumínio, para evitar a incidência da luz ambiente sobre as amostras. A medida do volume foi obtida diretamente na seringa. Esta pesquisa teve a aprovação do Comitê de Ética em Experimentação Animal do Instituto de Pesca, sob n⿳0 01/2017.

A fim de se evitar a contaminação do sêmen por ocasião da coleta, a região abdominal dos exemplares foi lavada com água doce, sendo imediatamente seca com toalhas de papel descartáveis antes de se iniciar a coleta. Após cada coleta, uma subamostra era observada em microscópio para avaliar se os espermatozoides estavam ativados (em função de contaminação por urina ou mesmo com resíduo de água) e, em caso positivo, a amostra era descartada. 
A avaliação da qualidade seminal foi realizada individualmente, antes da homogeneização do sêmen dos diversos machos, em partes iguais, para a realização dos experimentos de refrigeração (pool). Os parâmetros avaliados para cada amostra de sêmen individual e do pool foram: taxa de motilidade espermática (porcentagem de células da amostra que apresentam movimento progressivo), duração da motilidade espermática (duração da atividade de movimento celular, em segundos) e concentração espermática (número de espermatozoides/mL de sêmen) do sêmen fresco. A análise da motilidade e a da duração da motilidade espermáticas foram aferidas simultaneamente na mesma preparação, por um único técnico, usando um único campo focal escolhido aleatoriamente, com intensidade de luz mantida inalterada. Para a ativação do sêmen, foi utilizada uma taxa de diluição de 1:1, de acordo com Sanches et al. (2009), sendo obtida imediatamente após a adição da água marinha (salinidade 35$)$ ao sêmen $(15 \mu \mathrm{L}$ de sêmen: $15 \mu \mathrm{L}$ de água) e observada sob microscópio óptico, em ampliação de 200x.

A motilidade foi estimada registrando-se a porcentagem das células em movimento de deslocamento visualizadas no campo microscópico. A duração da motilidade foi cronometrada do início da ativação até o momento em que todas as células se tornaram imóveis no campo óptico (Sanches e Cerqueira, 2010). A concentração espermática foi avaliada por meio da contagem, sob microscópio (200x), das células espermáticas presentes em amostras de sêmen, previamente diluídas em solução tamponada de formol (5\%), preparadas em câmara hematimétrica de Neubauer $\left(1 \mathrm{~mm}^{3}\right)$.

Com as amostras de sêmen obtidas, foram realizados dois experimentos: refrigeração do sêmen com diferentes diluentes e refrigeração do sêmen em atmosfera modificada (100\% oxigênio). No primeiro experimento, foi analisado o efeito de diferentes soluções diluidoras na preservação da motilidade e da duração da motilidade do sêmen resfriado $\left(4^{\circ} \mathrm{C}\right)$ por até seis dias (144 horas). Foram empregadas três soluções iônicas anteriormente utilizadas com sucesso no congelamento do sêmen da garoupa-verdadeira (Sanches et al., 2009): diluente $\mathrm{A}(\mathrm{g} / \mathrm{L})=\mathrm{NaCl}, 7,89 ; \mathrm{KCl}, 1,19 ; \mathrm{CaCl}_{2}$, 0,$2 ; \mathrm{MgCl}_{2}, 0,4266 ; \mathrm{pH} 6,1 ; 158 \mathrm{mOsm}$; diluente $\mathrm{B}(\mathrm{g} / \mathrm{L})=\mathrm{NaCl}, 6,5 ; \mathrm{KCl}, 3,0 ; \mathrm{CaCl}_{2}, 0,3$;
$\mathrm{NaHCO}_{3}, 0,2 ; \mathrm{pH} 7,8 ; 157 \mathrm{mOsm}$; diluente C $(\mathrm{g} / \mathrm{L})=\mathrm{NaCl}, 7,89 ; \mathrm{KCl}, 1,19 ; \mathrm{CaCl}_{2}, 0,22$; $\mathrm{MgCl}_{2}, 0,72531 ; \mathrm{NaH}_{2} \mathrm{PO}_{4}, 0,0805 ; \mathrm{NaHCO}_{3}$, 0,$84 ; \mathrm{pH} 8,2 ; 172 \mathrm{mOsm}$.

Utilizaram-se amostras de sêmen de 10 indivíduos, em quantidades iguais, que apresentaram motilidade espermática média de $90 \%$, formando um pool. Foram realizados quatro tratamentos: controle (sêmen in natura, sem diluição); sêmen + diluente A; sêmen + diluente $\mathrm{B}$; sêmen + diluente $\mathrm{C}$. A proporção empregada de sêmen:diluente foi de 1:3 (volume de sêmen/volume do diluente), totalizando $1 \mathrm{~mL}$. Cada tratamento teve três repetições. As amostras (com volume sêmen + diluidor, totalizando $1 \mathrm{~mL}$ ) foram mantidas em tubos de ensaio de $5 \mathrm{~mL}$, devidamente identificadas e armazenadas em refrigerador $\left(4^{\circ} \mathrm{C}\right)$. A taxa e a duração da motilidade espermática de cada unidade experimental foram avaliadas após zero, $12,24,48,72$ e 144 horas de resfriamento a $4^{\circ} \mathrm{C}$. $\mathrm{O}$ momento $\mathrm{Oh}$ da avaliação foi o imediatamente após a diluição.

No segundo experimento, foi analisado o efeito de diferentes soluções diluidoras na preservação e na duração da motilidade do sêmen resfriado $\left(4^{\circ} \mathrm{C}\right)$ por até dois dias, utilizando-se atmosfera modificada (exclusivamente oxigênio). Empregaram-se as mesmas soluções diluentes do experimento anterior. Foi utilizada uma mistura de partes iguais de sêmen de 10 indivíduos (os mesmos do experimento anterior), com taxa de motilidade espermática média de $90 \%$. Realizaram-se quatro tratamentos: controle (sêmen in natura, sem diluição); sêmen + diluente $\mathrm{A}$; sêmen + diluente $\mathrm{B}$; sêmen + diluente C. A proporção empregada de sêmen:diluente foi de 1:3 (v/v). Cada tratamento teve três repetições. As amostras (com volume sêmen + diluidor, totalizando $1 \mathrm{~mL}$ ) foram mantidas em tubos de ensaio de $5 \mathrm{~mL}$, devidamente identificadas e armazenadas em refrigerador $\left(4^{\circ} \mathrm{C}\right)$. Todas as amostras foram seladas em um saco plástico (com capacidade de 60 litros), que foi inflado com oxigênio puro. A taxa e a duração da motilidade espermática de cada unidade experimental foram avaliadas após zero, 12,24 e 48 horas de resfriamento a $4^{\circ} \mathrm{C}$. O momento $\mathrm{Oh}$ da avaliação foi o imediatamente após a diluição. 
Para análise estatística dos experimentos, foi utilizado o SAS (Statistical..., 1990) (SAS INSTITUTE INC., 1990). Todos os dados foram testados para normalidade e homogeneidade das variâncias. Os dados das taxas de motilidade sofreram transformação angular, antes da análise estatística. A análise de variância foi efetuada pelo procedimento ANOVA. Posteriormente, para verificação de diferenças significativas, foi aplicado o teste de variação múltipla de Tukey. Valores de $\mathrm{P}<0,05$ foram considerados significantes.

\section{RESULTADOS E DISCUSSÃO}

O sêmen fresco apresentou volume médio de $0,36 \mathrm{~mL}$, com taxa de motilidade de $100 \%$ e valor médio da duração da motilidade espermática de 3060 segundos. A taxa e a duração da motilidade, a concentração e o volume espermáticos são parâmetros indicados para avaliação da qualidade do sêmen de uma espécie e de sua capacidade de fertilização (Alavi et al., 2004; Fan et al., 2014). A duração da motilidade média do sêmen fresco foi superior às encontradas para outras espécies de peixes marinhos que utilizam o mesmo habitat da garoupa-verdadeira. $\mathrm{O}$ sêmen do ariocó $L$. synagris apresentou motilidade superior a $90 \%$, permanecendo móvel por $137 \pm 20$ segundos (Sanches e Cerqueira, 2010), e o sêmen da cioba L. analis teve duração de motilidade de $148 \pm 29$ segundos (Sanches et al., 2013). Por outro lado, tempos elevados de motilidade espermática, superiores a 4.500 segundos, foram observados para a garoupa asiática Epinephelus malabaricus (Chao et al., 1992). Comparativamente, em peixes de água doce, a duração da motilidade é bem mais reduzida. Para o sêmen do curimbatá
Prochilodus brevis (Steindachnner, 1874), por exemplo, foram reportados apenas 63 segundos (Silva et al., 2014). A elevada duração da motilidade espermática no sêmen de serranídeos ainda não foi esclarecida, entretanto pode estar relacionada ao complexo processo reprodutivo, que envolve formação de haréns e extensas agregações reprodutivas em locais com expressivo fluxo de água (Seyboth et al., 2011; Carvalho et al., 2012).

A concentração espermática encontrada foi de $3,1 \times 10^{9}$ células $\mathrm{mL}^{-1}$, similar ao obtido com a mesma espécie por Sanches et al. (2009) e dentro do intervalo observado por Cabrita et al. (2009). Cabe ressaltar que a concentração obtida para $E$. marginatus no presente estudo pode estar relacionada ao fato de que se trata de exemplares em primeira maturação sexual, associado ao de que a concentração espermática pode variar ao longo da estação reprodutiva, com tendência a ser reduzida ao final do período (Aramli et al., 2014).

Para as análises de viabilidade espermática dos experimentos de refrigeração, foi considerada a premissa de Marques e Godinho (2004), que estabelece como limite para utilização do sêmen uma taxa de motilidade espermática de, no mínimo, 30\%. A motilidade espermática do sêmen fresco (sem adição de diluentes) diferiu $(\mathrm{P}<0,05)$ do sêmen com adição de diluentes, a partir de $24 \mathrm{~h}$ em diante, na temperatura de $4^{\circ} \mathrm{C}$. Outra observação importante foi a eficiência do diluente A em preservar a motilidade espermática (acima de 30\%) por até 144 horas, diferindo $(\mathrm{P}<0,05)$ dos diluentes $\mathrm{B}$ e $\mathrm{C}$ por períodos prolongados (Tab. 1).

Tabela 1. Taxa de motilidade (\%; média \pm desvio-padrão) do sêmen de Epinephelus marginatus ( $\mathrm{n}=10$ machos, três replicatas/tratamento) submetido à refrigeração a $4{ }^{\circ} \mathrm{C}$, diluído $1: 3(\mathrm{v} / \mathrm{v})$ em diferentes diluentes $^{(1)}$

\begin{tabular}{ccccccc}
\hline \multirow{2}{*}{ Diluentes $^{(2)}$} & \multicolumn{5}{c}{ Tempo de resfriamento $(\mathrm{h})$} \\
\cline { 2 - 6 } & 0 & 12 & 24 & 48 & 72 & 144 \\
\hline A (pH 6,1) & $100 \pm 0 \mathrm{aA}$ & $90 \pm 0 \mathrm{aA}$ & $90 \pm 0 \mathrm{aA}$ & $70 \pm 0 \mathrm{aB}$ & $70 \pm 5 \mathrm{aB}$ & $32 \pm 3 \mathrm{aC}$ \\
$\mathrm{B}(\mathrm{pH} 7,8)$ & $100 \pm 0 \mathrm{aA}$ & $90 \pm 0 \mathrm{aA}$ & $90 \pm 0 \mathrm{aA}$ & $60 \pm 0 \mathrm{aB}$ & $50 \pm 5 \mathrm{aB}$ & $5 \pm 1 \mathrm{bC}$ \\
C (pH 8,2) & $100 \pm 3 \mathrm{aA}$ & $90 \pm 0 \mathrm{aA}$ & $70 \pm 0 \mathrm{bB}$ & $20 \pm 0 \mathrm{bC}$ & $3 \pm 0 \mathrm{bD}$ & $0 \pm 0 \mathrm{cD}$ \\
Sêmen não diluído & $100 \pm 3 \mathrm{aA}$ & $40 \pm 3 \mathrm{bB}$ & $0 \pm 0 \mathrm{cC}$ & - & - & -
\end{tabular}

${ }^{(1)}$ Médias seguidas por letras distintas, minúsculas na coluna e maiúsculas na linha, diferem significativamente pelo teste de Tukey a $5 \%$ de probabilidade.

${ }^{(2)}$ Diluente A $(\mathrm{g} / \mathrm{L})=\mathrm{NaCl}, 7,89 ; \mathrm{KCl}, 1,19 ; \mathrm{CaCl}_{2}, 0,2 ; \mathrm{MgCl}_{2}, 0,4266 ; \mathrm{pH} 6,1 ; 158 \mathrm{mOsm}$; diluente $\mathrm{B}(\mathrm{g} / \mathrm{L})=\mathrm{NaCl}$,

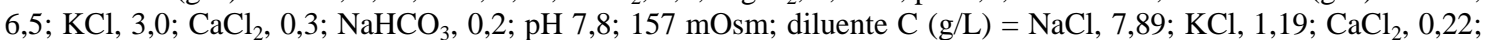
$\mathrm{MgCl}_{2}, 0,72531 ; \mathrm{NaH}_{2} \mathrm{PO}_{4}, 0,0805 ; \mathrm{NaHCO}_{3}, 0,84 ; \mathrm{pH} 8,2 ; 172 \mathrm{mOsm}$. 
Em relação ao tempo de duração da motilidade espermática, o sêmen fresco não suportou o rigor do processo de refrigeração, sendo observada uma queda brusca do parâmetro analisado em apenas 12 horas. $\mathrm{O}$ sêmen acrescido do diluente
A diferiu dos demais tratamentos $(\mathrm{P}<0,05)$ após 24h, não apresentando expressiva redução da duração da motilidade por até 144 horas de refrigeração (Tab. 2).

Tabela 2. Duração da motilidade (segundos; média \pm desvio-padrão) do sêmen de Epinephelus marginatus ( $\mathrm{n}=10$ machos, três replicatas/tratamento) submetido à refrigeração a $4^{\circ} \mathrm{C}$, diluído $1: 3$ (v/v) em diferentes diluentes ${ }^{(1)}$

\begin{tabular}{ccccccc}
\hline \multirow{2}{*}{ Diluentes $^{(2)}$} & \multicolumn{5}{c}{ Tempo de resfriamento (h) } \\
\cline { 2 - 6 } & 0 & 12 & 24 & 48 & 72 & 144 \\
\hline \multirow{2}{*}{$\mathrm{A}(\mathrm{pH} \mathrm{6,1)}$} & \multirow{2}{*}{$3060 \pm 600 \mathrm{aB}$} & $3800 \pm$ & $3400 \pm$ & $3200 \pm$ & $3100 \pm$ & $2900 \pm$ \\
& & $400 \mathrm{aA}$ & $300 \mathrm{aA}$ & $300 \mathrm{aA}$ & $200 \mathrm{aA}$ & $200 \mathrm{aB}$ \\
$\mathrm{B}(\mathrm{pH} \mathrm{7,8)}$ & \multirow{2}{*}{$3060 \pm 600 \mathrm{aB}$} & $3600 \pm$ & $3200 \pm$ & $2500 \pm$ & $2000 \pm$ & $1500 \pm$ \\
& & $400 \mathrm{aA}$ & $400 \mathrm{aA}$ & $300 \mathrm{bB}$ & $200 \mathrm{bC}$ & $200 \mathrm{bD}$ \\
$\mathrm{C}(\mathrm{pH} \mathrm{8,2)}$ & \multirow{2}{*}{$3060 \pm 600 \mathrm{aB}$} & $3600 \pm$ & $3000 \pm$ & $1200 \pm$ & $600 \pm$ & $0 \pm 0 \mathrm{cE}$ \\
& $400 \mathrm{aA}$ & $300 \mathrm{bB}$ & $200 \mathrm{cC}$ & $300 \mathrm{cD}$ & - \\
Sêmen não & \multirow{2}{*}{$3060 \pm 600 \mathrm{aA}$} & $600 \pm$ & $0 \pm 0 \mathrm{cC}$ & - & - & - \\
diluído & $200 \mathrm{bB}$ & & & & \\
\hline
\end{tabular}

${ }^{(1)}$ Médias seguidas por letras distintas, minúsculas na coluna e maiúsculas na linha, diferem significativamente pelo teste de Tukey a $5 \%$ de probabilidade.

${ }^{(2)}$ Diluente A $(\mathrm{g} / \mathrm{L})=\mathrm{NaCl}, 7,89 ; \mathrm{KCl}, 1,19 ; \mathrm{CaCl}_{2}, 0,2 ; \mathrm{MgCl}_{2}, 0,4266 ; \mathrm{pH} 6,1 ; 158 \mathrm{mOsm}$; diluente $\mathrm{B}(\mathrm{g} / \mathrm{L})=\mathrm{NaCl}$, 6,5; KCl, 3,0; $\mathrm{CaCl}_{2}, 0,3 ; \mathrm{NaHCO}_{3}, 0,2 ; \mathrm{pH} 7,8 ; 157 \mathrm{mOsm}$; diluente $\mathrm{C}(\mathrm{g} / \mathrm{L})=\mathrm{NaCl}, 7,89 ; \mathrm{KCl}, 1,19 ; \mathrm{CaCl}_{2}, 0,22$; $\mathrm{MgCl}_{2}, 0,72531 ; \mathrm{NaH}_{2} \mathrm{PO}_{4}, 0,0805 ; \mathrm{NaHCO}_{3}, 0,84 ; \mathrm{pH} 8,2 ; 172 \mathrm{mOsm}$.

Especificamente com o diluente A, foi possível manter a viabilidade espermática da garoupaverdadeira por até 144 horas de refrigeração. Intervalos de tempo inferiores aos obtidos neste estudo foram observados na refrigeração do sêmen de peixes de água doce, como a carpa Cyprinus carpio (Linnaeus, 1758) (11 horas) (Padua et al., 2012) e no sêmen do curimbatá $P$. brevis (21 horas) (Silva et al., 2014), contrastanto, entretanto, com o sêmen resfriado do baiacu Takifugu niphobles (Jordan e Snyder, 1901) por até 168 horas (Gallego et al., 2013). Independentemente do habitat, espermatozoides de diferentes espécies de peixes reagem de maneira distinta ao processo de refrigeração, sendo o metabolismo espermático a chave para a ampliação da viabilidade seminal durante $o$ processo de preservação pela redução da temperatura (Zietara et al., 2009).

Uma hipótese para o melhor desempenho do diluente A no processo de refrigeração do sêmen da garoupa-verdadeira seria a ausência de bicarbonato de sódio $\left(\mathrm{NaHCO}_{3}\right)$, que está presente no diluente $\mathrm{B}$ e, em maior quantidade, no C. Resultados positivos na refrigeração do sêmen da cioba (L. analis) com o mesmo diluente foram atribuídos ao efeito da dissociação do bicarbonato em meio aquoso, que resultaria em uma queda expressiva no $\mathrm{pH}$ intracelular dos espermatozoides e consequente efeito danoso na sobrevida espermática (Sanches e Cerqueira, 2011). O emprego de diluentes tamponados tem o objetivo de impedir que os metabólitos dos espermatozoides, acumulados no decorrer do processo de congelamento, causem mudanças no $\mathrm{pH}$ do sêmen, provocando efeitos deletérios às células espermáticas. Contrariamente ao processo de congelamento, que submete o sêmen a um rápido declínio de temperatura, seguido por inativação da atividade metabólica, o processo de refrigeração, entretanto, implica manutenção reduzida da atividade metabólica dos espermatozoides, o que poderia explicar o melhor desempenho do diluente A no processo de refrigeração.

Outro ponto a ser destacado foi o aumento da duração da motilidade espermática quando empregado o diluente A no processo de refrigeração do sêmen da garoupa-verdadeira. Diluentes com adequadas composições iônicas podem prolongar o tempo de motilidade espermática (Sanches et al., 2009). Aparentemente isso pode ser possível pela sustentação de um novo microambiente aos espermatozoides, distinto do ambiente fisiológico proporcionado pelo plasma seminal 
(Sanches e Cerqueira, 2010; Sanches e Cerqueira, 2011).

No experimento utilizando atmosfera modificada (100\% oxigênio), tanto a taxa de motilidade como a duração da motilidade espermática apresentaram uma expressiva queda, não sendo possível obter tempos de refrigeração superiores a 12 horas com resultados significativos de sobrevida espermática. O sêmen fresco diferiu $(\mathrm{P}<0,05)$ dos tratamentos com diferentes diluentes. Entretanto, não foram observadas diferenças entre os diferentes diluentes (Tab. 3 e 4). Resultados expressivos de $99 \%$ e $40 \%$ de motilidade espermática foram obtidos após cinco e 17 dias, respectivamente, para o sêmen do esturjão-do-atlântico Acipenser oxyrinchus (Mitchill, 1815) quando mantido sob refrigeração em tubos com atmosfera saturada de oxigênio
(Dilauro et al., 1994). No presente estudo, após 24 horas a taxa de motilidade foi significativamente mais alta em atmosfera normal, ocorrendo queda brusca da taxa de motilidade quando utilizada atmosfera modificada. Em estudos de refrigeração de sêmen utilizando atmosfera modificada, com duas espécies de lutjanídeos, não foram observados resultados positivos advindos da utilização de oxigênio, obtendo-se piores resultados comparativamente à utilização de atmosfera normal (Sanches e Cerqueira, 2010; Sanches e Cerqueira, 2011). Os autores reportaram que a oxidação do sêmen pelo contato direto do oxigênio poderia explicar os resultados observados. No presente estudo, não houve vantagem na utilização de uma atmosfera de $100 \%$ oxigênio durante o processo de refrigeração do sêmen de E. marginatus.

Tabela 3. Taxa de motilidade (\%; média \pm desvio-padrão) do sêmen de Epinephelus marginatus $(\mathrm{n}=10$ machos, três replicatas/tratamento) submetido à refrigeração a $4{ }^{\circ} \mathrm{C}$, em atmosfera modificada $(100 \%$ oxigênio), diluído $1: 3(\mathrm{v} / \mathrm{v})$ em diferentes diluentes ${ }^{(1)}$

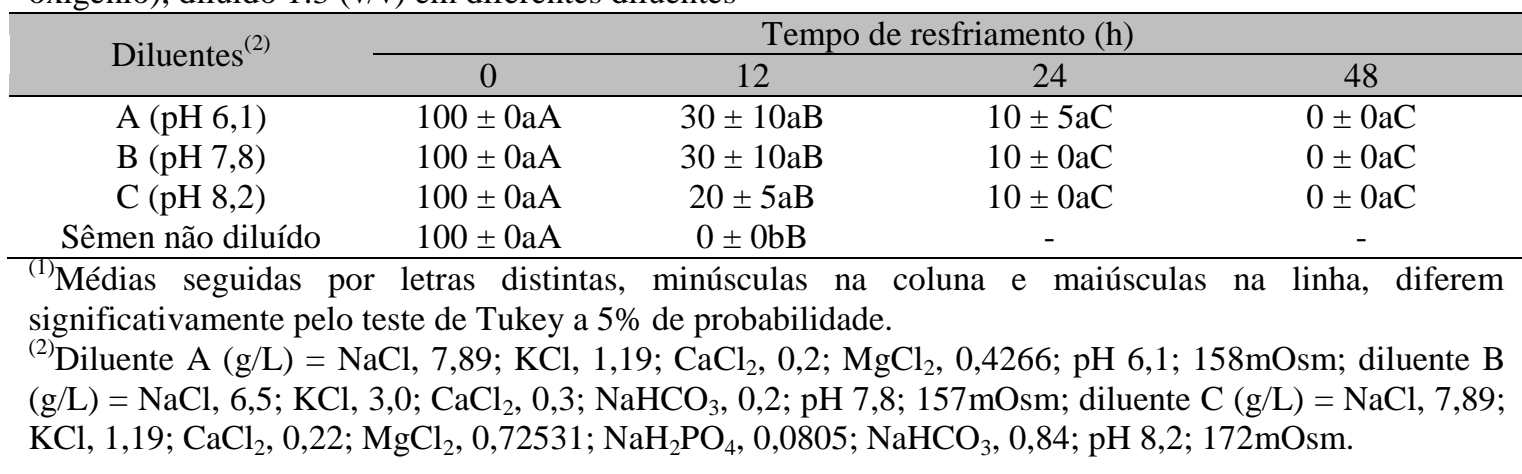

Tabela 4. Duração da motilidade (segundos; média \pm desvio-padrão) do sêmen de Epinephelus marginatus ( $\mathrm{n}=10$ machos, três replicatas/tratamento) submetido à refrigeração a $4^{\circ} \mathrm{C}$, em atmosfera modificada (100\% oxigênio), diluído 1:3 (v/v) em diferentes diluentes ${ }^{(1)}$

\begin{tabular}{ccccc}
\hline \multirow{2}{*}{ Diluente $^{(2)}$} & 0 & 12 & \multicolumn{4}{c}{ Tempo de resfriamento (h) } & 48 \\
\cline { 2 - 5 } & $3060 \pm 600 \mathrm{aA}$ & $2100 \pm 200 \mathrm{aB}$ & $1000 \pm 150 \mathrm{aC}$ & $0 \pm 0 \mathrm{aD}$ \\
$\mathrm{A}(\mathrm{pH} \mathrm{6,1)}$ & $3060 \pm 600 \mathrm{aA}$ & $2200 \pm 300 \mathrm{aB}$ & $1100 \pm 200 \mathrm{aC}$ & $0 \pm 0 \mathrm{aD}$ \\
$\mathrm{B}(\mathrm{pH} \mathrm{7,8)}$ & $3060 \pm 600 \mathrm{aA}$ & $2000 \pm 300 \mathrm{aB}$ & $1000 \pm 200 \mathrm{aC}$ & $0 \pm 0 \mathrm{aD}$ \\
C (pH 8,2) & $3060 \pm 600 \mathrm{aA}$ & $0 \pm 0 \mathrm{bB}$ & - & - \\
Sêmen não diluído &
\end{tabular}

${ }^{(1)}$ Médias seguidas por letras distintas, minúsculas na coluna e maiúsculas na linha, diferem significativamente pelo teste de Tukey a $5 \%$ de probabilidade.

${ }^{(2)}$ Diluente $\mathrm{A}(\mathrm{g} / \mathrm{L})=\mathrm{NaCl}, 7,89 ; \mathrm{KCl}, 1,19 ; \mathrm{CaCl}_{2}, 0,2 ; \mathrm{MgCl}_{2}, 0,4266 ; \mathrm{pH} 6,1 ; 158 \mathrm{mOsm}$; diluente B $(\mathrm{g} / \mathrm{L})=\mathrm{NaCl}, 6,5 ; \mathrm{KCl}, 3,0 ; \mathrm{CaCl}_{2}, 0,3 ; \mathrm{NaHCO}_{3}, 0,2 ; \mathrm{pH} 7,8 ; 157 \mathrm{mOsm}$; diluente $\mathrm{C}(\mathrm{g} / \mathrm{L})=\mathrm{NaCl}, 7,89$; $\mathrm{KCl}, 1,19 ; \mathrm{CaCl}_{2}, 0,22 ; \mathrm{MgCl}_{2}, 0,72531 ; \mathrm{NaH}_{2} \mathrm{PO}_{4}, 0,0805 ; \mathrm{NaHCO}_{3}, 0,84 ; \mathrm{pH} \mathrm{8,2;172mOsm}$.

A viabilidade econômica do cultivo da garoupaverdadeira é significativamente impactada pelo preço das formas jovens (Sanches et al., 2006).
Este foi o primeiro trabalho a obter sucesso na refrigeração do sêmen da garoupa-verdadeira, tornando possível a adoção dessa técnica como 
ferramenta reprodutiva, a fim de ampliar a oferta de formas jovens e promover o desenvolvimento do cultivo comercial dessa espécie. Os resultados demonstram que, para o processo de refrigeração, deve-se optar por um diluente que não apresente bicarbonato de sódio em sua composição e que a refrigeração deve ser feita preferencialmente em atmosfera normal. No intuito de otimizar os resultados na preservação da qualidade seminal, fica evidente a relevância de se considerar a influência dos componentes químicos dos diluentes no metabolismo espermático durante os processos de refrigeração e congelamento de sêmen. Por meio da utilização de técnicas de refrigeração de sêmen, como as testadas neste estudo, será possível avançar na adoção da reprodução dessa espécie em cativeiro como ferramenta de conservação. Considerandose a expressiva quantidade de espécies de peixes marinhos com potencial para cultivo e em situação de vulnerável ou ameaçada, a estratégia da refrigeração do sêmen pode proporcionar significativos avanços na conservação da ictiofauna brasileira.

\section{CONCLUSÕES}

A refrigeração do sêmen da garoupa-verdadeira E. marginatus é eficiente para a conservação de curto prazo, sendo possível, mediante o emprego do diluente A, manter por até 144 horas uma adequada qualidade espermática. Não há vantagens na utilização de atmosfera modificada (100\% oxigênio) na refrigeração do sêmen dessa espécie.

\section{REFERÊNCIAS}

ALAVI, S.M.H.; COSSON, J.; KARAMI, M.; AMIRI, B.M. Spermatozoa motility in the Persian sturgeon: effects of $\mathrm{pH}$, dilution rate, ions and osmolality. Reprod. Res., v.128, p.819828, 2004.

ARAMLI, S.; KALBASSI, M.R.; NAZARI, R.M. Monthly fluctuations during the breeding season of sperm density, volume, motility, and composition of seminal and coelomic fluid in broodfish of Persian sturgeon, Acipenser persicus Borodin, 1897. J. Appl. Ichthyol., v.30, p.261-266, 2014.
BILLARD, R.; COSSON, S.B.; NOVIERI M.; POURKAZEMI, M. Cryopreservation and shortterm storage of sturgeon: a review. Aquaculture, v.236, p.1-9, 2004.

CABRITA, E.; ENGROLA, S.; CONCEIÇÃO, L.E.C. et al. Successful cryopreservation of sperm from sex-reversed dusky grouper, Epinephelus marginatus. Aquaculture, v.287, p.152-157, 2009.

CARVALHO, M.O.X.; PAIVA, M.P.; FONTELES FILHO, A. Idade e crescimento da garoupa-verdadeira, Epinephelus marginatus (Lowe, 1834) (Pisces: Epinephelidae), no sudeste do Brasil. Arq. Ciênc. Mar, v.45, p.5-16, 2012.

CHAO, N.H.; TSAI, H.P.; LIAO. I.C. Short and long-term cryopreservation of sperm and sperm suspension of the grouper, Epinephelus malabaricus (Bloch and Schneider 1801). Asian Fish. Sci., v.5, p.103-116, 1992.

CUNHA, M.E.; RE, P.; QUENTAL-FERREIRA, H. et al. Larval and juvenile development of dusky grouper Epinephelus marginatus reared in mesocosms. J. Fish Biol., v.83, p.448-465, 2013.

DILAURO, M.N.; KRISE, W.F.; HENDRIX, M.A.; BAKER, S.E. Short term storage of Atlantic sturgeon sperm. Prog. Fish-Cult., v.56, p.143-144, 1994.

FAN, B.; LIU, X.C.; MENG, Z.N. et al. Cryopreservation of giant grouper Epinephelus lanceolatus (Bloch, 1790) sperm. J. Appl. Ichthyol., v.30, p.334-339, 2014.

GALLEGO, V.; PÉREZ, L.; ASTURIANO, J.F.; YOSHIDA, M. Study of pufferfish (Takifugu niphobles) sperm: development of methods for short-term storage, effects of different activation media and role of intracellular changes in $\mathrm{Ca}^{2+}$ and $\mathrm{K}^{+}$in the initiation of sperm motility. Aquaculture, v.414, p.82-91, 2013.

KERBER, C.E.; SILVA, H.K.A.; SANTOS, P.A.; SANCHES. E.G. Reproduction and larviculture of dusky grouper Epinephelus marginatus (Lowe, 1834) in Brazil. J. Agric. Sci. Tech., v.2, p.229-234, 2012.

KIRIYAKIT, A.; GALLARDO, W.G.; BART, A.N. Successful hybridization of groupers (Epinephelus coioides x Epinephelus lanceolatus) using cryopreserved sperm. Aquaculture, v.320, p.106-112, 2011. 
MARQUES, S.; GODINHO, H.P. Short-term cold storage of sperm from six tropical characiformes fishes. Braz. Arch. Biol. Tech., v.47, p.799-804, 2004.

OLIVEIRA, A.V.; VIVEIROS, A.T.M.; MARIA, A.N. et al. Sucesso do resfriamento e congelamento do sêmen de pirapitinga Brycon nattereri. Arq. Bras. Med. Vet. Zootec., v.59, p.1509-1515, 2007.

PADUA, N.H.; SHIMODA, E.; BARBOSA, P.S.; PEREIRA JUNIOR, G. Conservação do sêmen da carpa comum, Cyprinus carpio, variedade ornamental, por resfriamento. Acta Biomed. Bras., v.3, p.63-72, 2012.

RAMOS, F.M.; SANCHES, E.G.; FUJIMOTO, R.Y. et al. Growth of juvenile dusky grouper Epinephelus marginatus at three different diets. Bol. Inst. Pesca, v.38, p.81-88, 2012.

RED List of Threatened Species. Cambridge: IUCN, 2012. Available in: <http://www.iucnredlist.org>. Accessed in: 17 Jul. 2015.

ROUMBEDAKIS, K.; MARCHIORI, N.C.; PASETO, Á. et al. Parasite fauna of wild and cultured dusky-grouper Epinephelus marginatus (Lowe, 1834) from Ubatuba, Southeastern Brazil. Braz. J. Biol., v.73, p.871-878, 2013.

SALMITO-VANDERLEY, C.S.B.; VIEIRA, M.J.A.F.; LEITE, L.V. et al. Meios de congelação para conservação de sêmen de peixes da família characidae. Ciênc. Anim., v.22, p.255268,2012

SANCHES, E.G.; CERQUEIRA, V.R. Preservação de sêmen refrigerado de cioba com diluentes e atmosfera modificada. Pesqui. Agropecu. Bras., v.46, p.1673-1680, 2011.

SANCHES, E.G.; CERQUEIRA, V.R. Refrigeração do sêmen do ariocó Lutjanus synagris. Bol. Inst. Pesca, v.36, p.293-305, 2010.

SANCHES, E.G.; HENRIQUES, M.B.; FAGUNDES, L.; SILVA, A.A. Viabilidade econômica do cultivo da garoupa verdadeira (Epinephelus marginatus) em tanques-rede, região Sudeste do Brasil. Inf. Econom., v.36, p.15-25, 2006.
SANCHES, E.G.; OLIVEIRA, I.R.; SERRALHEIRO, P.C. Crioconservação do sêmen da garoupa-verdadera Epinephelus marginatus (Lowe, 1834) (Teleostei, Serranidae). Bol. Inst. Pesca, v.35, p.389-399, 2009.

SANCHES, E.G.; OLIVEIRA, I.R.; SERRALHEIRO, P.C.; CERQUEIRA, V.R. Cryopreservation of mutton snapper Lutjanus analis sperm. An. Acad. Bras. Ciênc., v.85, p.1083-1091, 2013.

SANCHES, E.G.; SILVA, F.C.; LEITE, J.R. et $a l$. A incorporação de óleo de peixe na dieta pode melhorar o desempenho da garoupa-verdadeira Epinephelus marginatus? Bol. Inst. Pesca, v.40, p.147-155, 2014.

SAS technical report. Version 6.11. Cary: SAS, 1990. 229p.

SEYBOTH, E.; CONDINI, M.V.; ALBUQUERQUE, C.Q. et al. Age, growth, and reproductive aspects of the dusky grouper Mycteroperca marginata (Actinopterygii: Epinephelidae) in a man-made rocky habitat in southern Brazil. Neotrop. Ichthyol., v.9, p.849856, 2011.

SILVA, A.C.; GALVÃO, J.A.S.; TEIXEIRA, E.G.; FARIAS, W.R.L. Caracterização e resfriamento do sêmen de curimbatá, Prochilodus brevis (Steindachnner, 1874). Rev. Bras. Hig. Sanid. Anim., v.8, 105-129, 2014.

SOUZA， R.S.R.; ANNUNCIACÃO, W.F.; LINS, S.M. et al. Can barber goby Elacatinus figaro control Neobenedenia melleni infections on dusky grouper Epinephelus marginatus? Aquacult. Res., v.45, p.619-628, 2014.

STATISTICAL analyses system. Versão 6.11 . Cari: SAS, 1990.

ZIETARA, M.S.; BIEGNIEWSKA, A.; RURANGWA, E. et al. Bioenergetics of fish spermatozoa during semen storage. Fish Physiol. Biochem., v.35, p.607-614, 2009. 